\title{
Insulin resistance in obese and lean women with polycystic ovarian syndrome
}

\section{Jayashree S., Shylaja P., Virupakshi Ajjammanavar*} Department of Obstetrics and Gynecology, JSS Medical College, JSS Academy of Higher Education and Research,
Mysore, Karnataka, India

Received: 07 December 2018

Accepted: 12 December 2018

\section{*Correspondence:}

Dr. Virupakshi Ajjammanavar,

E-mail: drvirupakshaa@yahoo.co.in

Copyright: (c) the author(s), publisher and licensee Medip Academy. This is an open-access article distributed under the terms of the Creative Commons Attribution Non-Commercial License, which permits unrestricted non-commercial use, distribution, and reproduction in any medium, provided the original work is properly cited.

\begin{abstract}
Background: According to NIH criteria for PCOS, the estimated prevalence of this disorder has been reported to range from $4 \%$ to $10 \%$ of women in their reproductive years, which designates PCOS as the most common endocrinopathy of women. Insulin resistance is common in PCOS and obesity contributes an additional component to insulin resistance in obese PCOS.

Methods: The study was a prospective study. One-hundred and twenty PCOS women were divided into two groups: Group $O$ - obese $(n=60)$ and Group L - lean (body mass index [BMI] cutoff $<23 \mathrm{~kg} / \mathrm{m} 2)$. Oral glucose tolerance test, serum fasting insulin and HOMA- IR were compared between these groups.

Results: Impaired glucose tolerance was seen in $33.3 \%$ of lean PCOS and 36.7\% of obese PCOS women. 5\% of lean PCOS and $10 \%$ of obese PCOS women had hyperinsulinemia. 38.3\% of lean PCOS and $51.7 \%$ of obese PCOS women had insulin resistance. But the differences were not statistically significant. However, HOMA-IR and fasting insulin values showed a significant positive correlation with BMI.

Conclusions: Both obese and lean women with PCOS are vulnerable to the problems of insulin resistance irrespective of BMI and insulin resistance shows a positive correlation with BMI.
\end{abstract}

Keywords: Fasting Insulin, HOMA-IR, Lean PCOS, Obese PCOS

\section{INTRODUCTION}

Polycystic ovarian syndrome (PCOS) was first reported by Stein and Leventhal in 1935. It is one of the most common endocrine disorders in reproductive age, affecting $5 \%$ to $10 \%$ of women worldwide. ${ }^{1}$ A high prevalence of up to $35 \%$ reported for the Indian women is of great concern. ${ }^{2}$ The 2003 Rotterdam Consensus Workshop concluded that PCOS is a syndrome of ovarian dysfunction along with cardinal features of hyperandrogenism and polycystic ovary (PCO) morphology. ${ }^{3}$

Achard and Thiers described the association between a disorder of carbohydrate metabolism and hyperandrogenism in 1921 and was called "the diabetes of bearded women". ${ }^{4}$

A study conducted on 100 lean women with PCOS found that $47 \%$ were insulin resistant. This shows the need for evaluating both obese and lean women with PCOS as its prevalence is high and to prevent the reproductive, metabolic and cardiovascular consequences. ${ }^{5}$ The presence of hyperinsulinemia in PCOS, independent of obesity, was confirmed by a number of groups worldwide.

This study was done to evaluate the biochemical markers of insulin resistance (fasting insulin, HOMA-IR (Homeostatic Model Assesment and Insulin Resistance) 
and oral glucose tolerance test) in obese (BMI>23) and lean women with PCOS $(\mathrm{BMI} \leq 23)$ and compare their values between them.

\section{METHODS}

This was a prospective study done on patients coming to OBG OPD of JSS hospital, Mysuru, satisfying inclusion and exclusion criteria.

One hundred and twenty patients between 14 and 40 years of age meeting at least two of the three parameters in Rotterdam criteria were included in the study.

\section{Inclusion criteria}

- Oligomenorrhea-Amenorrhoea

- Biochemical and/ or clinical hyperandrogenism

- $\quad$ PCO morphology in USG.

\section{Exclusion criteria}

- Patients with suspicion of androgen secreting tumour,

- Hyperprolactinemia, cushing's syndrome,

- Congenital adrenal secreting tumour,

- Thyroid dysfunction and pregnant women.

The patients were further divided into obese (Group O) and lean (Group L) PCOS groups. The body mass index (BMI) cut-off was taken as $\leq 23 \mathrm{~kg} / \mathrm{m} 2$ for lean PCOS. The patients were informed about the study and its confidential nature. A written and informed consent was taken.

This study was approved by and conducted according to the guidelines of the Institutional Ethical Committee. Detailed history was taken including presenting complaint(s), menstrual history, pattern of menstrual cycle, duration and extent of hair growth, voice changes, acne and weight gain.

Past history of diabetes mellitus, hypertension, thyroid disorders, hirsutism or galactorrhoea, treatment history for irregular cycles, diabetes, PCOS, infertility, baldness, excessive hair growth, treatment for acne or weight gain and family history of similar complaints were noted.

Height was measured to the nearest $0.1 \mathrm{~cm}$ using a stadiometer with the subject in the erect position, with her head held in Frankfurt horizontal plane.

Weight was measured to the nearest $0.1 \mathrm{~kg}$, without shoes and wearing light clothes, using an electronic digital weighing machine. BMI was calculated by the formula weight $(\mathrm{kg}) /$ [height $\left.\left(\mathrm{m}^{2}\right)\right]$.
Clinically insulin resistance was diagnosed subjectively by the presence of acanthosis nigricans. Hormonal evaluation was done after an overnight fasting using commercial kits in our laboratory. The hormonal profile included prolactin, thyroid-stimulating hormone and serum fasting insulin (FI).

Oral glucose tolerance test (OGTT) was done by obtaining fasting plasma glucose and plasma glucose 2 hours after $75 \mathrm{~g}$ oral glucose load. Samples were stored at $-20^{\circ} \mathrm{C}$ until the assay.

The glucose oxidase-peroxidase method was used for measurements of plasma glucose.

Serum fasting insulin level was measured using commercial kits and an electro-chemiluminiscence device. Insulin resistance (IR) was calculated by using the HOMA model [HOMA-IR=fasting serum insulin $(\mu \mathrm{U} / \mathrm{L}) \mathrm{x}$ fasting plasma glucose $(\mathrm{mmol} / \mathrm{L}) / 22.5]$.

HOMA-IR value more than 2.5 was considered as insulin resistance. Statistical methods applied were descriptive statistics, inferential statistics, Cramer's V test and Pearson correlation. $\mathrm{P}$ value $<0.05$ was considered statistically significant.

\section{RESULTS}

The present study was conducted in the Department of Obstetrics and Gynecology, J.S.S Medical College and Hospital, Mysore. One hundred and twenty patients diagnosed as having PCOS by Rotterdam criteria were divided into obese and lean PCOS patients.

In the present study $40 \%$ of lean PCOS patients were within the age group of 16- 20 years with a mean age of 22.5 years. $46.7 \%$ obese PCOS women were in the age group of 21-25 years with a mean age of 24 years.

There is a significant difference in the age group of lean women with PCOS compared to obese women, with more number of women being in the 16 to 20 years age group (Table 1).

Acanthosis nigricans, a clinical manifestation of insulin resistance, was seen in 3.3\% of lean PCOS patients and $10 \%$ of obese PCOS patients. There is no statistically significant difference between the two groups (Table 2).

Present study shows that $33.3 \%$ of lean PCOS and $36.7 \%$ of obese PCOS patients had impaired glucose tolerance. There is no significant difference between obese and lean women with respect to glucose intolerance. None of the women in both groups had type 2 diabetes mellitus (Table 3). 
Table 1: Age group of patients.

\begin{tabular}{|c|c|c|c|c|c|c|}
\hline & & & \multicolumn{2}{|c|}{ Group } & \multirow{2}{*}{ Total } & \multirow{2}{*}{ p value } \\
\hline & & & $\mathbf{L}$ & $\mathbf{O}$ & & \\
\hline \multirow{10}{*}{$\begin{array}{l}\text { Age } \\
\text { (years) }\end{array}$} & \multirow{2}{*}{$16-20$} & Count & 24 & 9 & 33 & 0.009 \\
\hline & & $\%$ within group & 40.0 & 15.0 & 27.5 & \\
\hline & \multirow{2}{*}{$21-25$} & Count & 20 & 28 & 48 & 0.248 \\
\hline & & $\%$ within group & 33.3 & 46.7 & 40.0 & \\
\hline & \multirow{2}{*}{$26-30$} & Count & 13 & 14 & 27 & 0.847 \\
\hline & & $\%$ within group & 21.7 & 23.3 & 22.5 & \\
\hline & \multirow{2}{*}{$31-35$} & Count & 3 & 6 & 9 & 0.317 \\
\hline & & $\%$ within group & 5.0 & 10.0 & 7.5 & \\
\hline & \multirow{2}{*}{$>35$} & Count & 0 & 3 & 3 & 1 \\
\hline & & $\%$ within group & 0.0 & 5.0 & 2.5 & \\
\hline \multirow{2}{*}{\multicolumn{2}{|c|}{ Total }} & Count & 60 & 60 & 120 & \\
\hline & & $\%$ within group & 100.0 & 100.0 & 100.0 & \\
\hline
\end{tabular}

Table 2: Acanthosis nigricans.

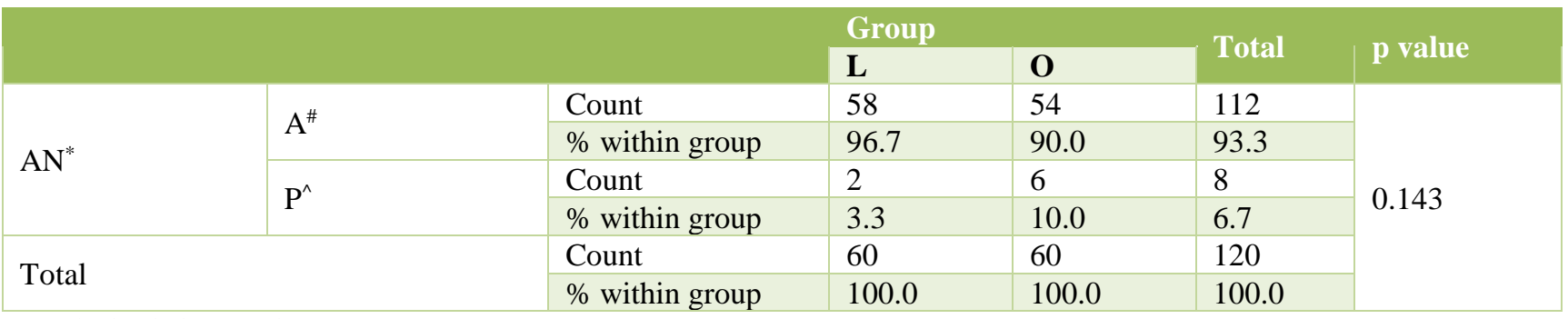

*Acanthosis Nigricans, \#Absent, $\wedge$ Present

Table 3: Oral glucose tolerance test.

\begin{tabular}{|c|c|c|c|c|c|c|}
\hline & & & \multicolumn{2}{|c|}{ Group } & \multirow{2}{*}{ Total } & \multirow{2}{*}{ p value } \\
\hline & & & $\mathbf{L}$ & $\mathbf{O}$ & & \\
\hline \multirow{4}{*}{ OGTT $^{*}$} & Normo1 & Count & 40 & 38 & 78 & \multirow{6}{*}{0.702} \\
\hline & Normal & $\%$ within group & 66.7 & 63.3 & 65.0 & \\
\hline & & Count & 20 & 22 & 42 & \\
\hline & Impaired & $\%$ within group & 33.3 & 36.7 & 35.0 & \\
\hline \multirow{2}{*}{\multicolumn{2}{|c|}{ Total }} & Count & 60 & 60 & 120 & \\
\hline & & $\%$ within group & 100.0 & 100.0 & 100.0 & \\
\hline
\end{tabular}

Table 4: Fasting insulin.

\begin{tabular}{|c|c|c|c|c|c|c|}
\hline & & & \multicolumn{2}{|c|}{ Group } & \multirow{2}{*}{ Total } & \multirow{2}{*}{ p value } \\
\hline & & & $\mathbf{L}$ & $\mathbf{O}$ & & \\
\hline \multirow{4}{*}{$\mathrm{FI}^{*}$} & Normal & Count & 57 & 54 & 111 & \multirow{6}{*}{0.298} \\
\hline & Normal & $\%$ & 95.0 & 90.0 & 92.5 & \\
\hline & & Count & 3 & 6 & 9 & \\
\hline & Abnormal & $\%$ & 5.0 & 10.0 & 7.5 & \\
\hline \multirow{2}{*}{\multicolumn{2}{|c|}{ Total }} & Count & 60 & 60 & 120 & \\
\hline & & $\%$ & 100.0 & 100.0 & 100.0 & \\
\hline
\end{tabular}

*Fasting insulin

Hyperinsulinemia was found in $5 \%$ of lean PCOS and $10 \%$ of obese PCOS patients. There is no statistically significant difference between both groups (Table 4). HOMA-IR of $\geq 2.5$, indicating insulin resistance, was found in $38.3 \%$ of lean PCOS and $51.7 \%$ of obese PCOS patients. The difference is not statistically significant (Table 5). There is a significant positive correlation of HOMA-IR and fasting insulin with BMI (Table 6). 
Table 5: HOMA-IR

\begin{tabular}{|c|c|c|c|c|c|c|}
\hline & & & \multicolumn{2}{|c|}{ Group } & \multirow{2}{*}{ Total } & \multirow{2}{*}{ p value } \\
\hline & & & $\mathbf{L}$ & $\mathbf{O}$ & & \\
\hline \multirow{4}{*}{ HOMA-IR ${ }^{*}$} & \multirow{2}{*}{$\geq 2.5$} & Count & 23 & 31 & 54 & \multirow{6}{*}{0.142} \\
\hline & & $\%$ within group & 38.3 & 51.7 & 45.0 & \\
\hline & \multirow{2}{*}{$<2.5$} & Count & 37 & 29 & 66 & \\
\hline & & $\%$ within group & 61.7 & 48.3 & 55.0 & \\
\hline \multirow{2}{*}{ Total } & & Count & 60 & 60 & 120 & \\
\hline & & $\%$ within group & 100.0 & 100.0 & 100.0 & \\
\hline
\end{tabular}

* Homeostatic model assessment and insulin resistance

Table 5: Correlation with BMI.

\begin{tabular}{|l|l|l|}
\hline \multirow{4}{*}{$\begin{array}{l}\text { HOMA- } \\
\text { IR }^{*}\end{array}$} & Pearson Correlation & 0.223 \\
\cline { 2 - 3 } & Significance (2-tailed) & 0.014 \\
\cline { 2 - 3 } & Number & 120 \\
\hline \multirow{3}{*}{$\begin{array}{l}\text { Fasting } \\
\text { Insulin }\end{array}$} & Pearson Correlation & 0.245 \\
\cline { 2 - 3 } & Significance (2-tailed) & 0.007 \\
\cline { 2 - 3 } & Number & 120 \\
\hline \multirow{2}{*}{ OGT $^{\#}$} & Pearson Correlation & 0.079 \\
\hline & Significance (2-tailed) & 0.391 \\
\hline & Number & 120 \\
\hline
\end{tabular}

\section{DISCUSSION}

PCOS constitutes a spectrum of symptoms starting from the early pre-pubertal years and continuing after menopause.

The phenotypic expression varies through time, depending on several internal (e.g. ovarian/ adrenal steroidogenesis, insulin resistance) and external factors (e.g. quality and quantity of food, exercise). ${ }^{6}$

Burghen et al in 1980 found that PCOS women had major metabolic as well as reproductive morbidity.

They reported that women with hyperandrogenic disorders like PCOS had basal and glucose stimulated hyperinsulinemia compared to weight matched controls suggesting presence of insulin resistance. ${ }^{7}$

The presence of hyperinsulinemia in PCOS, independent of obesity, was confirmed by a number of groups worldwide. Hughesdon reported in his study that hyperinsulinemia had an impact on ovarian morphology as well as function. ${ }^{8}$

Hyperinsulinemia exacerbates ovarian hyperandrogenism by

- Increasing $17 \alpha$-hydroxylase activity in theca cells a

- Promoting androstenedione and testosterone production; promoting LH- and IGF1-stimulated androgen production.
- Elevating free testosterone by decreasing the production of sex hormone binding globulin (SHBG).

Hyperandrogenism in turn results in arrest in the follicle development and anovulation. Metformin is the first line treatment for insulin resistance in PCOS.

In present study we evaluated two groups of PCOS patients, obese and lean. Relatively young population of PCOS women were enrolled in the present study with a majority of them presenting in the age group of 16-25 years.

Acanthosis nigricans (AN), a velvety, mossy, hyper pigmented skin disorder, which is a cutaneous manifestation of insulin resistance and correlates with the magnitude of peripheral insulin resistance was seen in $3.3 \%$ of lean PCOS patients and $10 \%$ of patients in obese group. In a study conducted by Akshaya et al also, the incidence of AN was similar in both the groups. ${ }^{9}$

In present study, $33.3 \%$ of lean PCOS and $36.7 \%$ of obese PCOS women had impaired glucose tolerance (IGT). None of the women in both the groups had type 2 diabetes mellitus.

There is no significant difference between obese and lean women with respect to glucose intolerance. In contrast to this, Gupta et al in their study found IGT in $12 \%$ and $8 \%$ of obese and non-obese group, respectively. ${ }^{10}$ Gambineri et al.,11 also reported similar results among PCOS subjects screened from Mediterranean region. ${ }^{11}$

A National Survey of Diabetes and IGT conducted in the year 2000 in six major cities of India showed a 13.1\% prevalence of IGT and 5\% prevalence of diabetes in the younger age group (20-40 years) of the general population. ${ }^{12}$ Majumdar et al. reported a higher prevalence of IGT and type-2 DM in obese PCOS women when compared with lean ones. ${ }^{13}$

Legro et al. reported $31 \%$ IGT and $7.5 \%$ type- 2 DM in obese PCOS patients and $10.3 \%$ IGT and $1.5 \%$ DM in non-obese PCOS which was 3 times that of general population. They found that PCOS women are at a 
significantly higher risk for IGT and type-2 DM at all weights and at a younger age. ${ }^{14}$ Ehrmann et al. found IGT in $35 \%$ of women with PCOS and diabetes in $10 \% .^{15}$ Elting et al. have reported a high prevalence of DM (4 times) and hypertension (2.5 times) in 346 lean PCOS as compared to general Dutch female population. ${ }^{16}$

When compared to the above-mentioned studies the mean age group of population was less than $30 \mathrm{yrs}$ in the present study. This might be the reason for none of our patients having Type $2 \mathrm{DM}$.

In the present study, $5 \%$ of lean PCOS and $10 \%$ of obese PCOS patients have hyperinsulinemia. There is no statistically significant difference between both groups. The mean fasting insulin (FI) of lean PCOS is $10 \pm 7.2$ $\mu \mathrm{U} / \mathrm{mL}$ and that of obese PCOS is $12 \pm 7 \mu \mathrm{U} / \mathrm{mL}$.

Gupta et al in their study showed the mean serum FI in Group I (obese PCOS) and Group II (non-obese PCOS) were $17.66 \pm 8.95 \mu \mathrm{U} / \mathrm{mL}$ and $13.54 \pm 6.40 \mu \mathrm{U} / \mathrm{mL}$, respectively. Both the PCOS groups had significantly higher FI levels than the controls, but within the PCOS groups, the levels were comparable.

Yildirim et al reported higher FI levels in non-obese PCOS women as compared to the normal controls and Silfen et al. reported more than 2-fold increase in FI levels and a significant decrease in estimations of insulin sensitivity in overweight as compared to normal weight adolescents with PCOS. ${ }^{17,18}$

It was also noted that $38.3 \%$ of lean PCOS and $51.7 \%$ of obese PCOS women had insulin resistance (IR) in present study. Gupta et al in their study noticed a higher IR between both obese and non-obese PCOS (44\% and 36\%) as compared to normal controls. ${ }^{10}$ Morales et al reported that insulin sensitivity reduced by $50 \%$ in lean PCOS when compared with normal controls. ${ }^{19}$

There was a further decrease in obese controls and a 2fold greater reduction in obese PCOS than in obese controls, suggesting that IR is a common feature in PCOS, and that obesity contributes an additional component to IR in obese PCOS. Silfen et al. reported a more than 2-fold increase in FI levels and a significant decrease in estimations of insulin sensitivity in overweight $(>25 \mathrm{~kg} / \mathrm{m} 2)$ as compared to normal 57 weight adolescents with PCOS. ${ }^{18}$

FG:FI ratio $<4.5$ was seen in $40 \%$ and $28 \%$ of obese and non-obese PCOS, respectively. Vrbíková et al. reported that lean PCOS women are not more insulin resistant when compared with healthy controls. ${ }^{20}$

In a recent 12-year follow-up study by conducted on 637 subjects by Ramezani et al, a higher prevalence of IR was noted among PCOS women. ${ }^{21}$
Both obese and lean women with PCOS are vulnerable to the problems of insulin resistance irrespective of BMI.

PCOS itself is a risk factor for insulin resistance and its long-term complications. When evaluating PCOS subjects, metabolic features and insulin resistance should also be evaluated irrespective of BMI to prevent long term complications.

\section{ACKNOWLEDGMENTS}

Authors would like to thank Dr. Sujatha MS, Professor and Head and other colleagues of the department of OBG, JSS Medical College and Hospital, Mysore in the conduct of the study.

Funding: No funding sources

Conflict of interest: None declared

Ethical approval: The study was approved by the Institutional Ethics Committee

\section{REFERENCES}

1. Bereck JS. Endocrine Disorders. In: Bereck JS, editor. Bereck and Novak's Gynecology. 15th ed. New Delhi: Wolters Kluwer Health (India )Pvt LtdLippincott Williams and Wilkins; 2012. p. 1066-99.

2. Krithika D Muralidhara, Prabha M Adhikari, Muralidhara D V. Overweight/Obesity and metabolic syndrome in women with polycystic ovary syndrome. Indian J Basic App Med Res. 2015;4(3):227-36.

3. Rotterdam ESHRE/ASRM-Sponsored PCOS consensus workshop group. Revised 2003 consensus on diagnostic criteria and long-term health risks related to polycystic ovary syndrome (PCOS). Hum Reprod Oxf Engl. 2004;19(1):41-7.

4. Archard C, Thiers J. Le virilisme pilaire et son association 1' insuffisance glycolytique(diabetes a femmes de barbe). Bull Acad Natl Med(Paris). 1921;(86):51-64.

5. Yildizhan B, Anik Ilhan G, Pekin T. The impact of insulin resistance on clinical, hormonal and metabolic parameters in lean women with polycystic ovary syndrome. J Obstet Gynaecol J Inst Obstet Gynaecol. 2016;36(7):893-6.

6. Livadas S, Diamanti-Kandarakis E. Polycystic ovary syndrome: definitions, phenotypes and diagnostic approach. Front Horm Res. 2013;40:1-21.

7. Burghen GA, Givens JR, Kitabchi AE. Correlation of hyperandrogenism with hyperinsulinism in polycystic ovarian disease. J Clin Endocrinol Metab. 1980;50(1):113-6.

8. Hughesdon PE. Morphology and morphogenesis of the Stein-Leventhal ovary and of so-called 'hyperthecosis'. Obstet Gynecol Surv. 1982;37(2):59-77.

9. Akshaya S, Bhattacharya ratnaboli. Comparitive study of clinical profile of lean and obese polycystic 
ovary syndrome women. Int $\mathbf{J}$ Reprod Contracept Obstet Gynecol.2017;5(8):2530-3.

10. Gupta N, Radhakrishnan G, S V M, A G R. Comparison of metabolic and endocrinal parameters in obese and nonobese women of polycystic ovarian syndrome with normal controls. Fertil Sci Res. 2015;2(1):19.

11. Gambineri A, Pelusi C, Manicardi E, Vicennati V, Cacciari M, Morselli-Labate AM, et al. Glucose intolerance in a large cohort of mediterranean women with polycystic ovary syndrome: phenotype and associated factors. Diab. 2004;53(9):2353-8.

12. Snehalatha C, Ramchandran A, Kapur A, Vijay V. Age-specific prevalence and risk associations for impaired glucose tolerance in urban southern Indian population. J Assoc Physicians India. 2003;51:766-9.

13. Majumdar A, Singh TA. Comparison of clinical features and health manifestations in lean vs. obese Indian women with polycystic ovarian syndrome. J Hum Reprod Sci. 2009;2(1):12-7.

14. Legro RS, Kunselman AR, Dodson WC, Dunaif A. Prevalence and predictors of risk for type 2 diabetes mellitus and impaired glucose tolerance in polycystic ovary syndrome: a prospective, controlled study in 254 affected women. J Clin Endocrinol Metab. 1999;84(1):165-9.

15. Ehrmann DA, Barnes RB, Rosenfield RL, Cavaghan MK, Imperial J. Prevalence of impaired glucose tolerance and diabetes in women with polycystic ovary syndrome. Diabetes Care. 1999;22(1):141-6.

16. Elting MW, Korsen TJ, Bezemer PD, Schoemaker J. Prevalence of diabetes mellitus, hypertension and cardiac complaints in a follow-up study of a Dutch PCOS population. Hum Reprod Oxf Engl. 2001;16(3):556-60.
17. Yildirim B, Sabir N, Kaleli B. Relation of intraabdominal fat distribution to metabolic disorders in nonobese patients with polycystic ovary syndrome. Fertil Steril. 2003;79(6):1358-64.

18. Silfen ME, Denburg MR, Manibo AM, Lobo RA, Jaffe R, Ferin M, et al. Early endocrine, metabolic, and sonographic characteristics of polycystic ovary syndrome (PCOS): comparison between nonobese and obese adolescents. J Clin Endocrinol Metab. 2003;88(10):4682-8.

19. Morales AJ, Laughlin GA, Bützow T, Maheshwari H, Baumann G, Yen SS. Insulin, somatotropic, and luteinizing hormone axes in lean and obese women with polycystic ovary syndrome: common and distinct features. J Clin Endocrinol Metab. 1996;81(8):2854-64.

20. Vrbíková J, Vondra K, Cibula D, Dvoráková K, Stanická S, Srámková D, et al. Metabolic syndrome in young Czech women with polycystic ovary syndrome. Hum Reprod Oxf Engl. 2005;20(12):3328-32.

21. Ramezani Tehrani F, Montazeri SA, Hosseinpanah F, Cheraghi L, Erfani H, Tohidi M, et al. Trend of Cardio-Metabolic Risk Factors in Polycystic Ovary Syndrome: A Population-Based Prospective Cohort Study. PloS One. 2015;10(9):e0137609.

Cite this article as: Jayashree $S$, Shylaja $P$,

Ajjammanavar V. Insulin resistance in obese and lean women with polycystic ovarian syndrome. Int J Reprod Contracept Obstet Gynecol 2019;8:63-8. 